\title{
How does hippocampus contribute to working memory processing?
}

\author{
Marcin Leszczynski* \\ Department of Epileptology, University of Bonn, Bonn, Germany \\ *Correspondence: leszczynski.marcin@gmail.com
}

\section{A commentary on}

Constructing realistic engrams: poststimulus activity of hippocampus and dorsal striatum predicts subsequent episodic memory

by Ben-Yakov, Y., and Dudai, Y. (2011). J. Neurosci. 31, 9032-9042. doi: 10.1523/ jneurosci.0702-11.2011

The hippocampus was traditionally viewed as an area supporting declarative long-term memory (LTM). However, an increasing amount of evidence points beyond the LTM hypothesis showing that the hippocampus is involved during processing of relational memory (Eichenbaum, 2004) or spatial and spatiotemporal discontiguity (Staresina and Davachi, 2009). It has been also repeatedly found to get recruited during working memory (WM) maintenance of novel items (Ranganath and D'Esposito, 2001; Axmacher et al., 2007, 2010b; Fuentemilla et al., 2010; Poch et al., 2011). Intracranial EEG recordings in the hippocampus of epilepsy patients showed that maintenance of increasing WM load was associated with elevated negativity of evoked response potentials (Axmacher et al., 2007) and increase in theta/gamma phase-amplitude coupling (Axmacher et al., 2010b). Furthermore, it has been shown that shortly after offset of a sensory (to-be-remembered) input its neural representation is being replayed in medial temporal lobe (MTL; Fuentemilla et al., 2010; Poch et al., 2011). In this context a particularly interesting finding has been recently reported in the Journal of Neuroscience by Ben-Yakov and Dudai (2011).

In an elegant functional magnetic resonance imaging (fMRI) experiments, Ben-Yakov and Dudai (2011) identified brain regions which are activated immediately after the offset of complex stimulus sequences (movie clips) and correlate with subsequent recall performance. In the Experiment 1, the authors report bilateral hippocampus activity starting immediately after stimulus presentation. Importantly the analyses were time-locked to the offset of sensory input, tagging post-perceptual processing in the absence of sensory stimulation. The activation was found to increase for subsequently remembered over forgotten content. In Experiment 2 Ben-Yakov and Dudai (2011) used movies of different durations to examine whether onset of the activation varied with the stimulus duration. As predicted, the delayed hippocampal BOLD response was related to the offset of sensory stimuli rather than the duration of the presented material itself. Finally, in Experiment 3 the authors provided additional data supporting their hypothesis by comparing activation elicited by movie clips with different lengths but the same beginning. Together, Ben-Yakov and Dudai (2011) report that delayed bilateral activation of hippocampus and caudate nucleus, time-locked to stimulus offset was predictive for subsequent recall. The authors proposed that this post-stimulus activity reflects offline (triggered after perceiving an event) encoding, the process of binding experiences into cohesive units and registering it into memory.

I would like to suggest that the results might reflect WM maintenance rather than offline encoding supporting the hypothesis that WM maintenance involves hippocampus. WM maintenance is associated with stimulus offset and it serves to update previously perceived, yet perceptually unavailable items. Similarly, the concept of offline encoding as presented by the authors assumes a mechanism that operates at the offset of sensory stimuli and reflects the process of registering presented stimuli to existing knowledge. Additionally, they point out the relevance for LTM recall. This enumeration describes well the process of WM maintenance that has been linked to the hippocampus (Axmacher et al., 2007; Poch et al., 2011), and has been found to trigger a process of replay that is time-locked to the offset of sensory stimuli (Fuentemilla et al., 2010; Poch et al., 2011). WM maintenance has been also shown to interfere with simultaneous LTM encoding (Axmacher et al., 2010a). Furthermore, the MTL activation during WM maintenance predicts LTM encoding (Schon et al., 2004). This suggests an overlap between the WM and LTM processing. It is unclear whether this overlap reflects interaction or rather temporal co-activation of the two independent memory systems. The former require a cross-talk between WM and LTM. The latter suggests independent activation of WM and LTM processes triggered at the stimulus offset. In either case the alternative WM hypothesis for the offset activity being related to maintenance rather than encoding. In sum, WM maintenance is an LTM recall relevant mechanism that keeps previously perceived representation vivid for memory registration. This is achieved by periodical replay of information in the hippocampus with a rhythmic activity in the theta range.

When seeing the movie clip participants were told to pay attention to the gist. Given they were instructed to view the stimuli attentively and that they were going to be tested upon the watched movies it is fair to assume that participants focused their attention on the representation of a gist. Such a focus has been known to persist in maintenance in a process of strengthening sensory input that is no longer provided. It is known to operate for basic sensory features (Awh et al., 2000) as well as for more abstract object representations (Lepsien and Nobre, 2007). This further supports the notion of maintenance as a possible explanation of the hippocampus activity. Additionally, given that participants were instructed to encode each movie according to its gist rather than detailed information 
it is likely that the amount of information which they needed to remember from a single movie did not exceed WM span. This makes it possible that at movie offset participants were maintaining in WM a gist in order to register it to LTM justifying the expectation of a replay in hippocampus locked to the offset of stimuli.

Together, the results of Ben-Yakov and Dudai (2011) offers an interesting corroboration of the hypothesis that hippocampus is involved in WM processing. The idea that the observed activation reflects WM maintenance rather than offline encoding offers not only parsimony but also predictions. One prediction is that hippocampal activation locked to the stimulus offset consists of replayed representation of the sensory input, which might be tested using multi-variate pattern classification methods (Fuentemilla et al., 2010; Poch et al., 2011). Furthermore, the replay may not be continuous but rather occur in a periodic manner fluctuating with theta rhythm. Additionally, the replay should be categoryspecific corresponding to the gist of the movie that has just been viewed.

\section{ACKNOWLEDGMENTS}

I would like to thank Dr. Nikolai Axmacher and Dr. Jürgen Fell for helpful comments.

\section{REFERENCES}

Awh, E., Anllo-Vento, L., and Hillyard, S. A. (2000). The role of spatial selective attention in working memory for locations: evidence from event-related potentials. J. Cogn. Neurosci. 12, 840-847.

Axmacher, N., Lenz, S., Haupt, S., Elger, C. E., and Fell, J. (2010a). Electrophysiological signature of working and long-term memory interaction in the human hippocampus. Eur. J. Neurosci. 1, 177-188.

Axmacher, N., Henseler, M. M., Jensen, O., Weinreich, I., Elger, C. E., and Fell, J. (2010b). Cross-frequency coupling supports multi-item working memory in the human hippocampus. Proc. Natl. Acad. Sci. U.S.A. 107, 3228-3233.

Axmacher, N., Mormann, F., Fernández, G., Cohen, M. X. Elger, C. E., and Fell, J. (2007). Sustained neural activity patterns during working memory in the human medial temporal lobe. J. Neurosci. 27, 7807-7816.

Ben-Yakov, Y., and Dudai, Y. (2011). Constructing realistic engrams: poststimulus activity of hippocampus and dorsal striatum predicts subsequent episodic memory. J. Neurosci. 31, 9032-9042.

Eichenbaum, H. (2004). Hippocampus: cognitive processes and neural representations that underlie declarative memory. Neuron 44, 109-120.

Fuentemilla, L., Penny, W. D., Cashdollar, N., Bunzeck, N. and Düzel, E. (2010). Theta-coupled periodic replay in working memory. Curr. Biol. 20, 607-612.
Lepsien, J., and Nobre, A. C. (2007). Attentional modulation of object representations in working memory. Cereb. Cortex 17, 2072-2083.

Poch, C., Fuentemilla, L., Barnes, G. R., and Düzel, E. (2011). Hippocampal theta-phase modulation of replay correlates with configural-relational short-term memory performance. J. Neurosci. 31, 7038-7042.

Ranganath, C., and D'Esposito, M. (2001). Medial temporal lobe activity associated with active maintenance of novel information. Neuron 31 865-873.

Schon, K., Hasselmo, M. E., Lopresti, M. L., Tricarico, M. D., and Stern, C. E. (2004). Persistence of parahippocampal representation in the absence of stimulus input enhances long-term encoding: a functional magnetic resonance imaging study of subsequent memory after a delayed match-to-sample task. $J$. Neurosci. 24, 11088-11097.

Staresina, B. P., and Davachi, L. (2009). Mind the gap: binding experiences across space and time in the human hippocampus. Neuron 63, 267-276.

Received: 19September 2011; accepted: 30 November 2011; published online: 19 December 2011.

Citation: Leszczynski M (2011) How does hippocampus contribute to working memory processing? Front. Hum. Neurosci. 5:168. doi: 10.3389/fnhum.2011.00168

Copyright ( $(2011$ Leszczynski. This is an open-access article distributed under the terms of the Creative Commons Attribution Non Commercial License, which permits non-commercial use, distribution, and reproduction in other forums, provided the original authors and source are credited. 